\title{
Spin-phonon induced magnetic order in the kagome ice
}

\author{
F. A. Gómez Albarracín, ${ }^{*}$ D. C. Cabra, H. D. Rosales, and G. L. Rossini \\ IFLP - Departamento de Física, Universidad Nacional de La Plata, C.C. 67, 1900 La Plata, Argentina
}

(Received 19 June 2013; revised manuscript received 27 September 2013; published 20 November 2013)

\begin{abstract}
We study the effects of lattice deformations on the kagome spin ice, with Ising spins coupled by nearest-neighbor exchange and long-range dipolar interactions, in the presence of in-plane magnetic fields. We describe the lattice energy according to the Einstein model, where each site distortion is treated independently. Upon integration of lattice degrees of freedom, effective quadratic spin interactions arise. Classical Monte Carlo simulations are performed on the resulting model, retaining up to third neighbor interactions, under different directions of the magnetic field. We find that as the effect of the deformation is increased, a rich plateau structure appears in the magnetization curves.
\end{abstract}

DOI: 10.1103/PhysRevB.88.184421

PACS number(s): $75.40 . \mathrm{Mg}, 75.10 . \mathrm{Hk}$

\section{INTRODUCTION}

Spin ice systems ${ }^{1}$ have been the object of intense study in the last couple of decades. These materials are an experimental evidence of high magnetic frustration, showing a residual lowtemperature entropy and magnetic disorder. Some compound examples are $\mathrm{Ho}_{2} \mathrm{Ti}_{2} \mathrm{O}_{7}$ (Refs. 2 and 3) and $\mathrm{Dy}_{2} \mathrm{Ti}_{2} \mathrm{O}_{7}$ (Refs. 4 and 5), where the rare-earth ions form a lattice of cornersharing tetrahedra (pyrochlore lattice).

Due to intense crystal fields, spin degrees of freedom are locally projected onto a well-isolated Ising doublet ground state, pointing either in or out from the tetrahedra centers, meaning that the spins can be modeled by local Ising variables. Moreover, the transverse components of the gyromagnetic tensor vanish due to structural reasons, thus decoupling transverse field components and allowing for a classical treatment of these Ising variables. ${ }^{6,7}$ The interplay between antiferromagnetic nearest-neighbor exchange couplings and strong dipolar interactions in these materials leads to a highly frustrated and degenerate ground state satisfying the so-called ice rules: ${ }^{8,9}$ two spins in and two spins out of each tetrahedron.

The magnetization curves of the pyrochlore systems $\mathrm{Ho}_{2} \mathrm{Ti}_{2} \mathrm{O}_{7}$ (Ref. 10) and $\mathrm{Dy}_{2} \mathrm{Ti}_{2} \mathrm{O}_{7}$ (Ref. 11) show a wellknown plateau at $1 / 3$ of saturation under an external magnetic field in the $\langle 111\rangle$ direction. ${ }^{12,13}$ Above this plateau the apical spins are completely aligned with the external magnetic field, which suggests that the relevant physics stems from the transverse kagome layers. This paper is motivated by recent experiments ${ }^{14,15}$ which have identified a plateaulike feature above 1/3 magnetization, when the magnetic field is slightly tilted with respect to the $\langle 111\rangle$ direction. This feature is not described by the standard model for pyrochlore spin ice ${ }^{1}$ with only first-neighbors exchange couplings. Even with the inclusion of further neighbor couplings ${ }^{16}$ and careful treatment of the long-range dipolar interactions, ${ }^{17}$ this issue remains elusive. On the other hand, it is well known that spin lattice couplings lead to a variety of phenomena, such as the appearance of a plateau structure in the magnetization curves. ${ }^{18-20}$ Motivated by the need to understand the physics above the $1 / 3$ plateau, we have considered the influence of the phonon degrees of freedom in the magnetic properties of the pyrochlore. One should recall that for large spins the effects of the phonons appear generically enhanced by an $S^{2}$ factor.
In the high-field regime (above the pyrochlore $1 / 3$ plateau) the apical spins are aligned with the field and the remaining physics could be described, in a first approximation, by decoupled kagome planes. In fact, the three-dimensional character of the pyrochlore system could be retained by considering interlayer dipolar couplings, but this is out of the scope of the present paper. On the other hand, our model could be relevant in the study of artificial spin-ice systems. In the present work we consider a kagome ice model under the influence of a magnetic field along the plane which mimics the tilting of the magnetic field in the experiments. We include the effects of phonons, which induce a rich plateau structure.

The paper is organized as follows: In Sec. II we introduce the kagome ice model for the effective description of pyrochlore spins in active layers in the regime of interest, including nearest-neighbor exchange and dipolar interactions, and the spin interactions induced by lattice fluctuations. In Sec. III we present magnetization curves under in-plane magnetic fields, obtained by simulated annealing. Several plateaus and their magnetic ordered structure are described. Section IV is devoted to discussion and conclusions.

\section{KAGOME ICE MODEL}

We are interested in the description of Ising pyrochlore systems in the regime where apical spin magnetization is saturated. As mentioned in the Introduction, once the apical spins of the tetrahedra are aligned with the external magnetic field, the remaining spins lie on kagome planes. As a first step, we do not consider the out-of (kagome)-plane components of the spins and consider a kagome ice planar model, ${ }^{21}$ i.e., local spin directions point towards or outward from the center of the triangles of the kagome unit cell. Each site $i$ allocates a local Ising spin $\vec{S}_{i}=S \sigma_{i} \hat{e}_{i}$, where $S$ is the spin magnitude, $\sigma_{i}= \pm 1$ is the Ising variable $(+1$ being "in" and -1 "out"), and $\hat{e}_{i}$ is the local reference direction $(1,2,3$ in Fig. 1). This simplified model may also be relevant to artificial Permalloy arrays with kagome geometry, ${ }^{22,23}$ with the proviso that array deformations should have the same energy scale as magnetic interactions. The standard model for pyrochlore spin ice $^{1}$ includes exchange antiferromagnetic interactions only for nearest neighbors $\left(N^{(1)}\right)$ and long-range dipolar interactions; 


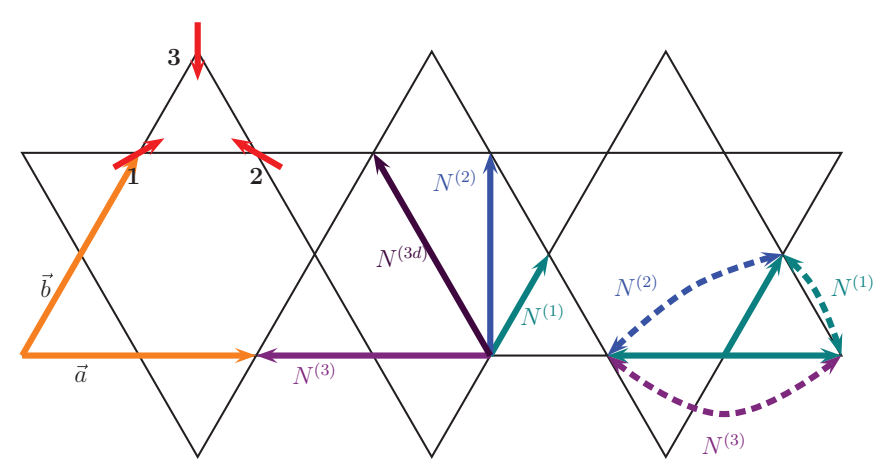

FIG. 1. (Color online) Kagome lattice. In red, the local reference directions for Ising spins on each site $\left(\sigma_{i}=+1\right.$ is referred to as "in"). First-, second-, and third-nearest neighbors are indicated. On the right, effective interactions arising from $\vec{F}_{i j}^{(1)}$ (green arrows) and bridged by a site $i$ are shown by dashed lines.

the Hamiltonian on the regular kagome lattice then reads

$$
\begin{aligned}
H_{0}= & J_{0} \sum_{\langle i j\rangle^{(\mathrm{l})}} \vec{S}_{i} \cdot \vec{S}_{j} \\
& +D a^{3} \sum_{i \neq j}\left[\frac{\vec{S}_{i} \cdot \vec{S}_{j}}{\left(r_{i j}^{0}\right)^{3}}-\frac{3\left(\vec{S}_{i} \cdot \hat{r}_{i j}\right)\left(\vec{S}_{j} \cdot \hat{r}_{i j}\right)}{\left(r_{i j}^{0}\right)^{3}}\right] \\
& -\vec{h} \cdot \sum_{i} \vec{S}_{i},
\end{aligned}
$$

where $J_{0}$ is the antiferromagnetic $N^{(1)}$ exchange interaction coupling, $D$ is the strength of the dipolar coupling, $a$ the distance between nearest neighbors, $r_{i j}^{0}$ the distance between any pair of spins at sites $i$ and $j$, and $\hat{r}_{i j}$ is the unit vector from site $i$ to site $j . \vec{h}=h_{x} \hat{x}+h_{y} \hat{y}$ is the external magnetic field in the kagome plane, $\hat{x}$ being perpendicular to one of the spin directions (say 3 in Fig. 1) and $\hat{y}$ parallel to it. In the description of pyrochlore in a magnetic field mentioned in the Introduction, a small $\vec{h}$ would account for a deviation of the magnetic field with respect to the $\langle 111\rangle$ direction. The out-of-plane component of the spins could be easily included, together with the $\langle 111\rangle$ component of the magnetic field and first-neighbor coupling of kagome plane spins to frozen apical spins. This generalization, together with its relation to magnetization experiments in dysprosium pyrochlore, ${ }^{4,5}$ will be considered in future work.

In order to include the effects of lattice deformations in the magnetic order, we introduce deformations $\vec{u}_{i}$ in the site positions

$$
\vec{r}_{i}=\vec{r}_{i}^{0}+\vec{u}_{i},
$$

so that the distance between sites $i$ and $j$ is distorted from the regular lattice. At first order

$$
r_{i j}=\left|\vec{r}_{j}-\vec{r}_{i}\right| \approx r_{i j}^{0}+\hat{r}_{i j} \cdot\left(\vec{u}_{j}-\vec{u}_{i}\right) .
$$

The effect of the deformations in the exchange interaction is taken at linear order to be isotropic,

$$
J\left(r_{i j}\right) \approx J_{0}\left[1-\alpha \hat{r}_{i j} \cdot\left(\vec{u}_{j}-\vec{u}_{i}\right)\right],
$$

where $\alpha=-\left.\frac{1}{J_{0}} \frac{\partial J}{\partial r_{i j}}\right|_{r_{i j}=r_{i j}^{0}}>0$ is the scalar spin-phonon coupling constant. Correspondingly, corrections to dipolar inter- actions are considered at first order by varying distances in the second line of Eq. (1).

We treat the elastic degrees of freedom in the adiabatic limit, assuming large ion masses, which is appropriate in the case of $\mathrm{Dy}_{2} \mathrm{Ti}_{2} \mathrm{O}_{7}$. There are different models to describe the energy cost of lattice deformations. ${ }^{24}$ One of them is the bond phonon model ${ }^{25}$ describing acoustic modes where the elastic energy depends on bond length deformation but each bond is allowed to independently expand or contract (ignoring geometrical constraints), i.e., variables $\delta \vec{r}_{i j}=\vec{u}_{j}-\vec{u}_{i}$ are independent. We have explored the effect of these modes in the effective description and we trivially observe that they just lead to a constant shift in the energy. The other standard choice is the Einstein phonon model, ${ }^{26}$ describing optical modes. It is known that, at the nearest-neighbor level, the kagome dispersion relations have a flatband of zero modes; however, the presence of further neighbor couplings should lift these modes, making the Einstein model a reliable approximation. The elastic energy is quadratic on each site displacement and truly independent deformations $\vec{u}_{i}$ can be exactly integrated. The spin-phonon Hamiltonian reads

$$
H=H_{0}+\sum_{i}\left(\frac{K}{2}\left(\vec{u}_{i}\right)^{2}+\vec{u}_{i} \cdot \sum_{j \neq i} \vec{F}_{i j}\right) .
$$

Here $\vec{F}_{i j}$ collects all terms proportional to $\vec{u}_{i}$ and containing $\sigma_{i} \sigma_{j}$, arising from a first-order expansion of the variation of $H_{0}$ with lattice distortions (see the Appendix). In this case, phonon degrees of freedom are easily integrated to yield an effective Hamiltonian for the magnetic degrees of freedom at a given temperature. In order to discuss the validity of this integration and subsequent approximations, we set $J_{0}$ as the energy scale and $a$ as the length scale to introduce dimensionless parameters $d=\frac{D}{J_{0}}$ for the dipolar interaction strength $k=\frac{K a^{2}}{J_{0}}$ for the phonon stiffness, and $\lambda=a \alpha$ for the linear spin-phonon coupling.

The standard Gaussian integration over elastic thermal fluctuations in the presence of linear interactions assumes that both the width and mean of the thermal distribution of displacements given by $e^{-\beta H}$ are much smaller than the lattice distance $a$. This requires for the phonon stiffness that $k \gg \frac{k_{B} T}{J_{0}}$, which is valid at low enough temperature $T$, and that the interaction factors $\vec{F}_{i j}$ satisfy

$$
\left|\sum_{j \neq i} \vec{F}_{i j}\right| \ll k J_{0} .
$$

The explicit expression for $\vec{F}_{i j}$ at first neighbors includes the spin-phonon coupling and a dipolar term

$$
\vec{F}_{i j^{(1)}}=\frac{J_{0} S^{2}}{a}\left(-\frac{\lambda}{2}+\frac{21}{4} d\right) \hat{r}_{i j} \sigma_{i} \sigma_{j}
$$

while the longer range $\vec{F}_{i j^{(n)}}$ only includes dipolar terms and decays with distance as $1 / r_{i j}^{4}$. In particular, for second neighbors one finds

$$
\vec{F}_{i j^{(2)}}=-\frac{J_{0} S^{2}}{a} \frac{5}{12} d \hat{r}_{i j} \sigma_{i} \sigma_{j},
$$


and for third neighbors the numerical factor decays to $3 / 16$. Taking $\vec{F}_{i j^{(1)}}$ as the significative contribution, Eq. (6) requires

$$
S^{2}\left|-\frac{\lambda}{2}+\frac{21}{4} d\right| \ll k .
$$

After Gaussian integration the effective Hamiltonian reads

$$
H_{\text {eff }}=H_{0}-\sum_{i} \frac{a^{2}}{2 k J_{0}} \sum_{j, k \text { neighbors of } i} \vec{F}_{i j} \cdot \vec{F}_{i k} .
$$

Notice that $\vec{F}_{i j} \cdot \vec{F}_{i k}$ contains $\sigma_{i}^{2}=1$, so it is proportional to $\sigma_{j} \sigma_{k},{ }^{27}$ thus effective corrections to interactions between Ising spins at sites $j, k$ arise from the summation of $\vec{F}_{i j} \cdot \vec{F}_{i k}$ terms in Eq. (10) over all $i \neq j, k$. Notice also that we depict each contribution to the interactions between sites $j$ and $k$ as bridged by a site $i$. In this sense, when both factors $\vec{F}_{i j}$ and $\vec{F}_{i k}$ refer to nearest neighbors, the site $i$ bridges interactions between first, second, and third neighbors as shown in Fig. 1. Longerrange factors $\vec{F}_{i j^{(n)}}$ give rise to increasingly long-range effective interactions, with smaller couplings.

In order to tailor a tractable effective model, we proceed to truncate the range of neighbors $N(i)$ in Eq. (10). To this aim we compare in detail the effective corrections to first-neighbors effective Ising coupling arising only from $\vec{F}_{i j^{(1)}}$ with those including second-range factors $\vec{F}_{i j^{(2)}}$. The relative weight of such second-range corrections is negligible if

$$
\frac{5 \sqrt{3}}{6} d \ll\left|\frac{21}{4} d-\frac{1}{2} \lambda\right| .
$$

Longer-range contributions from $\vec{F}_{i j^{(n)}}$ are even smaller, due to the dipolar decay. Condition (11) is largely satisfied in the case of dysprosium pyrochlore, where $\lambda$ can be estimated to be of order $20,{ }^{28}$ whereas values of $d \approx 1 / 3^{1}$ as we consider below. Thus we neglect $\vec{F}_{i j^{(n)}}$ for $n \geqslant 2$ in what follows.

Regarding the original long-range dipolar interactions in $H_{0}$, it is known that a truncation is more sensible in the kagome lattice than in the pyrochlore lattice because of lower dimensionality. ${ }^{22}$ According to the range of effective corrections kept, we also truncate long-range dipolar interactions, retaining up to third neighbors.

The truncated effective Hamiltonian finally reads

$$
\begin{aligned}
\frac{H_{\mathrm{eff}}}{J_{0} S^{2}}= & {\left[J_{\mathrm{eff}}^{(1)} \sum_{\langle i j\rangle^{(1)}} \sigma_{i} \sigma_{j}+J_{\mathrm{eff}}^{(2)} \sum_{\langle i j\rangle^{(2)}} \sigma_{i} \sigma_{j}\right.} \\
& \left.+J_{\mathrm{eff}}^{(3)} \sum_{\langle i j\rangle^{(3)}} \sigma_{i} \sigma_{j}+J_{\mathrm{eff}}^{(3 d)} \sum_{\langle i j\rangle^{(3 d)}} \sigma_{i} \sigma_{j}\right] \\
& -\sum_{i} \sigma_{i}\left[\tilde{h}_{x}\left(\hat{e}_{i}\right)_{x}+\tilde{h}_{y}\left(\hat{e}_{i}\right)_{y}\right],
\end{aligned}
$$

where $\langle i j\rangle^{(1)},\langle i j\rangle^{(2)},\langle i j\rangle^{(3)}$, and $\langle i j\rangle^{(3 d)}$ refer to first $N^{(1)}$, second $N^{(2)}$, third along triangle edges $N^{(3)}$, and third along hexagon diagonals $N^{(3 d)}$ neighbors, respectively, as depicted in Fig. 1. The dimensionless magnetic field $\tilde{h}_{\alpha}$ is defined as $h_{\alpha} /\left(J_{0} S\right)$, with $\alpha=x, y$. The effective couplings are given by

$$
\begin{aligned}
J_{\text {eff }}^{(1)} & =-\frac{1}{2}+\frac{7}{4} d-\delta, \\
J_{\text {eff }}^{(2)} & =-\frac{5}{12 \sqrt{3}} d+\delta, \\
J_{\text {eff }}^{(3 d)} & =\frac{1}{8} d, \\
J_{\text {eff }}^{(3)} & =-\frac{5}{32} d+2 \delta,
\end{aligned}
$$

with $\delta=\frac{S^{2}}{4 k}\left(-\frac{\lambda}{2}+\frac{21}{4} d\right)^{2}$. In a realistic pyrochlore setting, the corrections to dipolar interactions at second and third neighbors generated by phonon degrees of freedom could be related to those included as exchange interactions in Ref. 16 for tuning diffuse elastic neutron scattering data in dysprosium titanate.

Notice that after having integrated the phonon degrees of freedom, the effective model to be analyzed is a pure Ising model, but the inclusion of phonons ends up being a crucial ingredient in establishing the relative strength of the various couplings. This is at the root of the physics discussed in the present paper.

\section{MONTE CARLO SIMULATIONS - RESULTS AND DISCUSSION}

We analyze the effective Hamiltonian in Eq. (12) in a regime where exchange and first-order dipolar interactions compete, yielding a frustrating Ising interaction $\left(J_{\text {eff }}^{(1)}>0\right)$, and where the effective parameters $J_{\text {eff }}^{(1)}$ and $J_{\text {eff }}^{(2)}$ have the same order of magnitude (strong frustration regime). To this aim we consider $d \simeq 1 / 3$ for the rest of the paper. For these values of $d$, all considered interactions indeed compete. It should be noticed that $J_{\text {eff }}^{(1)}$ is positive up to $\delta=0.06$, thus favoring frustration, and $J_{\text {eff }}^{(2)}$ remains negative up to $\delta \approx 0.08$ [see Eq. (13)].

We performed Monte Carlo simulations for kagome lattices of $N=3 \times L^{2}$ sites, with $L=12,18, \ldots, 36$, by conventional single-spin flip, ${ }^{29}$ plus implementation of a tempering algorithm (annealing technique), ${ }^{30}$ lowering the temperature in a $T_{i+1}=T_{i} \times 0.9$ scheme down to the lowest value, $T=0.0042 J_{0} S^{2} / k_{B}$. At every magnetic field and temperature we discarded $2 \times 10^{6}$ Monte Carlo steps (MCSs) for initial relaxation and data were collected during subsequent $4 \times 10^{6}$ MCSs. Monte Carlo runs for the same parameters with different seeds gave no significant variations; thus no error bars are reported in average magnetization data.

We focus on the low-temperature phase diagram as a function of the single parameter $\delta$ in the presence of an external magnetic field. To determine the different phases we computed the normalized magnetization under magnetic fields applied along the $\hat{x}$ and $\hat{y}$ direction, defined as

$$
M_{\alpha}=\frac{1}{M_{\alpha, s}} \sum_{i=1}^{N}\left(\vec{S}_{i}\right)_{\alpha}
$$

where $\alpha=x, y$ and $M_{\alpha, s}$ is the saturation magnetization along $\alpha$. 


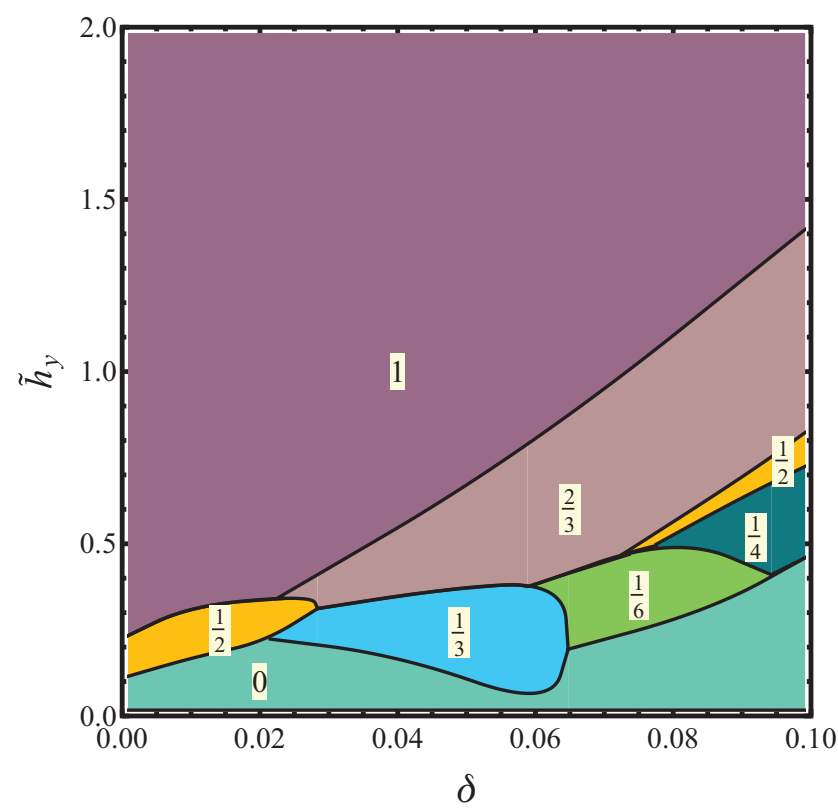

FIG. 2. (Color online) Phase diagram $h_{y}$ vs $\delta$ for a $3 \times L^{2}$ ( $L=30$ ) site kagome lattice. The numbers indicate the normalized magnetization of the plateaus.

\section{A. Magnetic field parallel to one of the spins}

We first study the equilibrium average magnetization $\left\langle M_{y}\right\rangle$ under fields $\vec{h}=h_{y} \hat{y}$, from zero to saturation $\left(\left\langle M_{y}\right\rangle=1\right)$. We performed simulations for $d=0.32$ and $\delta$ from 0 to 0.1 , where the condition in Eq. (6) is satisfied, in steps of 0.001 . The resulting phase diagram is shown in Fig. 2. It should be stressed that in all the observed plateau phases a single domain ordered structure is found, lifting the typical residual low-temperature entropy of pure spin-ice systems.

We find that for $\delta=0$, which corresponds to phonon stiffness $k \rightarrow \infty$ (no lattice deformations), there are two plateaus, for $\left\langle M_{y}\right\rangle=0$ and $\left\langle M_{y}\right\rangle=1 / 2$ [Figs. 3(a)-3(c)]. These plateaus, present by the only effect of dipolar interactions, are stable, lowering the value of $k$ (allowing for lattice deformations) up to $\delta \simeq 0.02$, where $J_{\text {eff }}^{(1)} \simeq J_{\text {eff }}^{(3 d)} \simeq 2\left|J_{2}^{\text {eff }}\right| / 3$ and $J_{\text {eff }}^{(3)} \simeq 0$ changes sign from negative to positive, adding frustration to the system.

For larger $\delta(>0.03)$, there is a transition: the $1 / 2$ plateau "splits" into two plateaus at $\left\langle M_{y}\right\rangle=1 / 3$ and $\left\langle M_{y}\right\rangle=2 / 3$, which widen as $\delta$ increases [Figs. 3(d)-3(f)]. Close to $\delta=$ $0.06, J_{\text {eff }}^{(1)} \simeq 0$ and the effective system is dominated by the third neighbors couplings with $J_{\text {eff }}^{(3 d)} \simeq J_{\text {eff }}^{(3)} / 2$ and $\left|J_{\text {eff }}^{(2)}\right|<$ $J_{\text {eff }}^{(3)} / 4$. We recall that for $\delta>0.06, J_{\text {eff }}^{(1)}$ is negative and does not favor frustration; therefore the lattice configurations no longer satisfy the kagome ice rules. In this region, where the system forms frustrated antiferromagnetic sublattices coupled by $J_{\text {eff }}^{(3 d)}$ and $J_{\text {eff }}^{(3)}$, with smaller $J_{\text {eff }}^{(1)}, J_{\text {eff }}^{(2)}$, a series of plateaus appear: $\left\langle M_{y}\right\rangle=1 / 6$ for $0.065<\delta<0.085$, and $\left\langle M_{y}\right\rangle=1 / 4$ and $\left\langle M_{y}\right\rangle=1 / 2$ again for $0.085<\delta$.

The local spin configurations at the different plateaus show magnetic order, which we describe by repetition of magnetic unit cells. These are sketched for each plateau in Figs. 4 and 5.
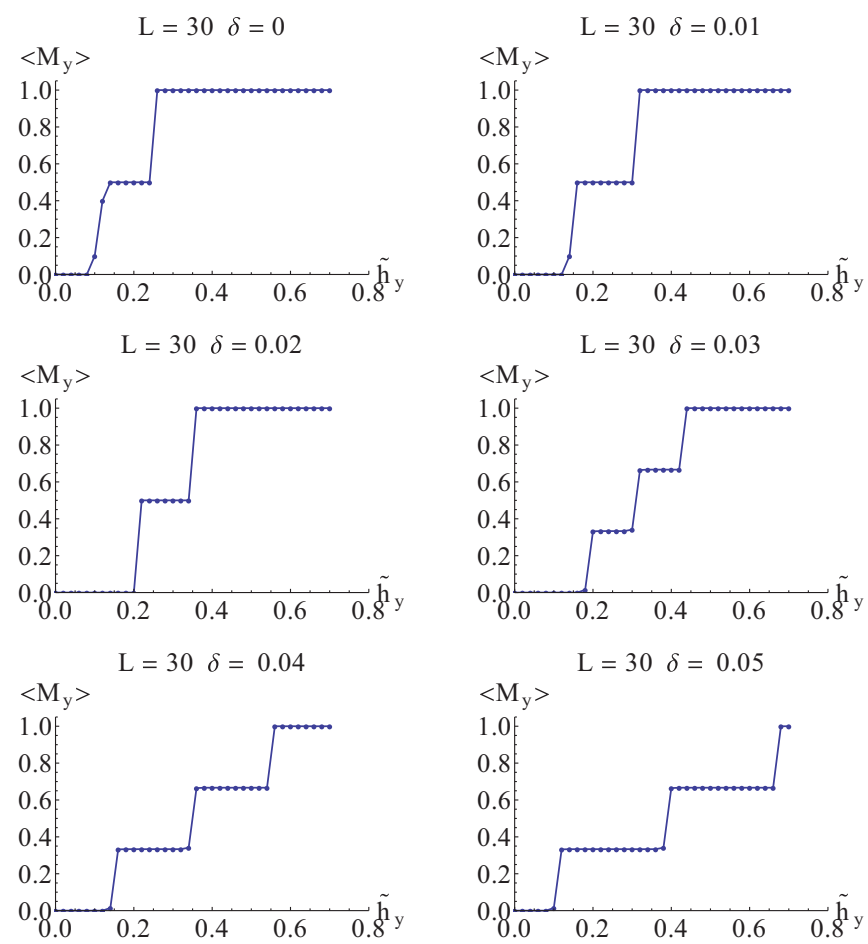

FIG. 3. (Color online) Average magnetization per spin vs $h_{y}$ for a $3 \times L^{2}(L=30)$ site kagome lattice.

At $\left\langle M_{y}\right\rangle=0,\left\langle M_{y}\right\rangle=1 / 3$, and $\left\langle M_{y}\right\rangle=2 / 3$ the magnetic pattern is obtained by translations along the $\{\vec{a}+\vec{b}, 2 \vec{a}-\vec{b}\}$ vectors (depicted in Fig. 1) of a nine-site magnetic unit cell consisting of three kagome unit-cell triangles (sites 1, 2, 3) forming a star. In Fig. 4(a) we show in detail such tiling at $\left\langle M_{y}\right\rangle=0$, where the $\sigma_{i}=+1$ are arranged in closed hexagons inside each star, surrounded by $\sigma_{i}=-1$. In Fig. 4(b) the content of the magnetic unit cell is shown for the following plateaus: at $\left\langle M_{y}\right\rangle=1 / 3$ one of the spins in a site 3 of every hexagon flips, so the $\sigma_{i}=+1$ form a ' $\mathrm{C}$,' while in the $\left\langle M_{y}\right\rangle=2 / 3$ plateau another $\sigma_{3}=+1$ flips to -1 . At this point, every spin in every site 3 is aligned with the external magnetic field. Finally, the lattice reaches its saturation configuration where the star consists of three identical triangles.

In contrast to the plateaus described before by a nine-site magnetic unit cell, the rest of the plateaus exhibit different size magnetic unit cells. In the $\left\langle M_{y}\right\rangle=1 / 2$ regime shown in Fig. 2 for small $\delta$, the six-site magnetic unit cell is a combination of two reflected kagome unit-cell triangles with $\sigma_{3}=-1$ and $\sigma_{1}=-\sigma_{2}$, as shown in Fig. 4(c).

We recall that all of the lattice triangles in the plateaus mentioned above satisfy kagomelike ice rules: spins pointing two-in, one-out, or the other way around. Notice that we are not keeping track here of the apical spins in the pyrochlore spin ice motivating our analysis; above the $1 / 3$ plateau, with aligned apical spins, three-dimensional spin-ice rules would impose a constraint, allowing only two-in, one-out spins in kagome triangles. For larger values of $\delta$ the magnetic configurations break the kagome ice rules and we find, at $\left\langle M_{y}\right\rangle=1 / 6$, there is a 36-site magnetic unit cell, at $\left\langle M_{y}\right\rangle=1 / 4$ a 18 -site magnetic 
(a)

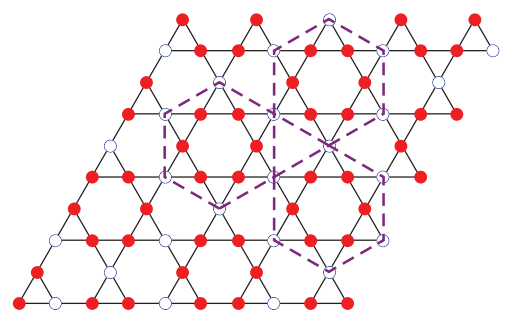

(b)

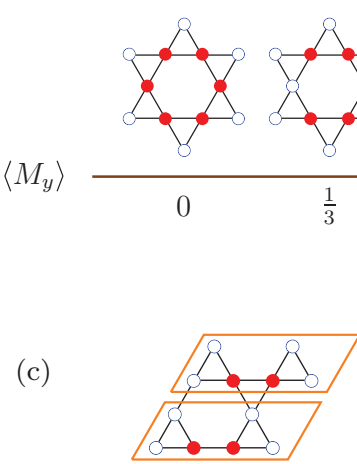

$\left\langle M_{y}\right\rangle=\frac{1}{2}$

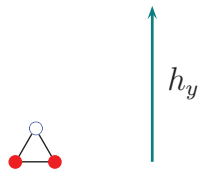

$\left\langle M_{y}\right\rangle=1$
FIG. 4. (Color online) Magnetic unit cells in the kagome lattice in different magnetization plateaus for $h_{x}=0$. In each elementary triangle (see Fig. 1, triangle with sites 1-2-3), open blue circles indicate $\sigma_{i}=-1$ (out) and full red circles $\sigma_{i}=+1$ (in). (a) $\left\langle M_{y}\right\rangle=0$ tiling and its magnetic unit cell; (b) magnetic unit cell for $\left\langle M_{y}\right\rangle=$ $0,1 / 3$, and $2 / 3$. The $\left\langle M_{y}\right\rangle=1$ case has a smaller unit cell as shown in (c). At the $1 / 3$ plateau both the figure shown or its specular reflection can be found; (c) $\left\langle M_{y}\right\rangle=0$ and 1 .

unit cell, and finally, at $\left\langle M_{y}\right\rangle=1 / 2$ a 12-site magnetic unit cell. All these magnetic unit cells are shown in Fig. 5.

It is interesting to notice that for any of the plateaus shown in Fig. 2 the $\mathbb{Z}_{2}$ reflection symmetry of the system in the presence of $h_{y}$ is not broken $\left(\left\langle M_{x}\right\rangle=0\right)$.

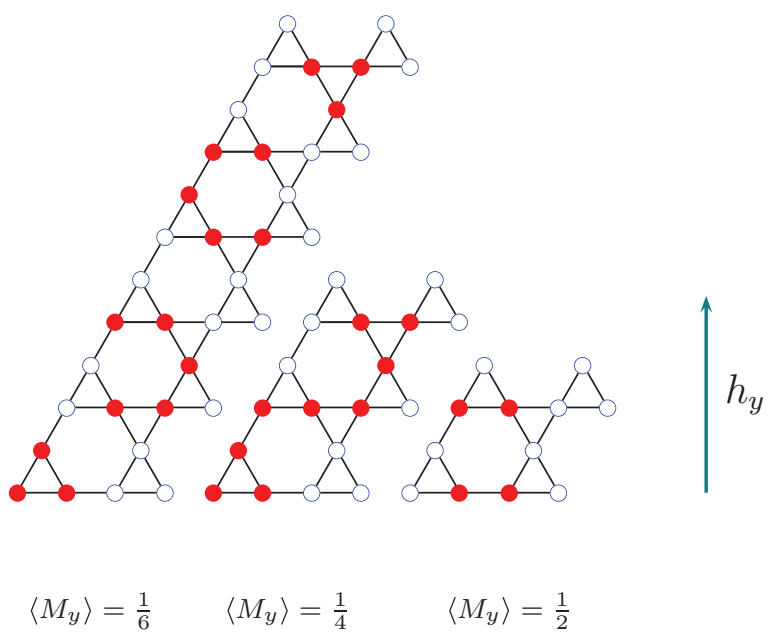

FIG. 5. (Color online) Magnetic unit cells in the kagome lattice in different magnetization plateaus in the large- $\delta$ regime. The $1 / 6$, $1 / 3$, and $1 / 2$ plateaus at the large- $\delta$ regime.

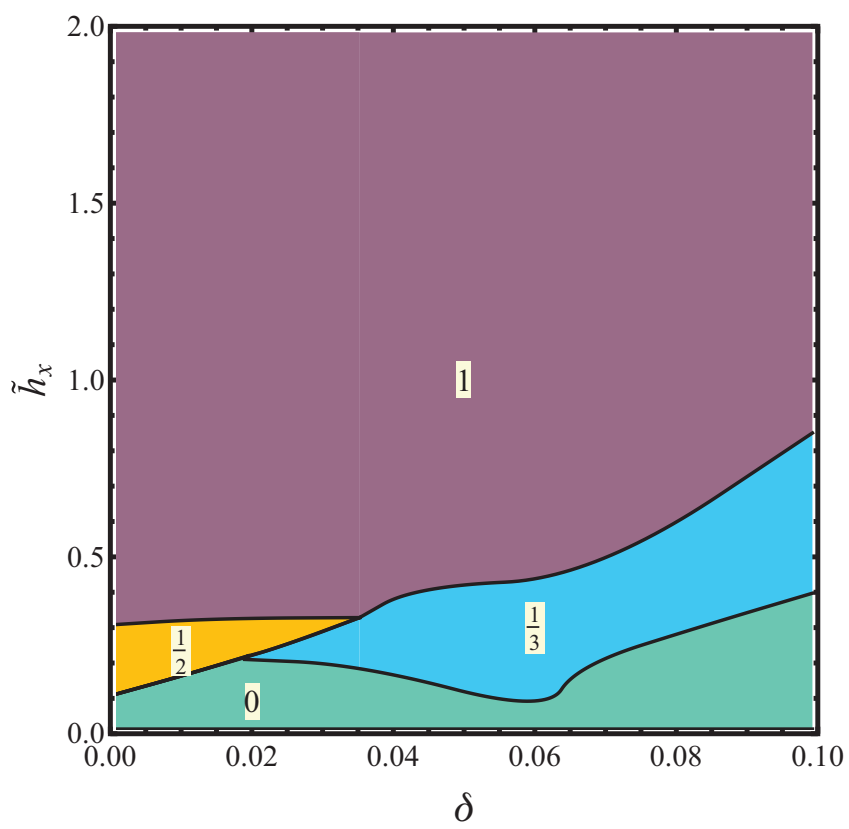

FIG. 6. (Color online) Phase diagram $h_{x}$ vs $\delta$. The numbers show the normalized magnetization of the plateaus. Only the $\left\langle M_{x}\right\rangle=1 / 3$ plateau is induced by lattice deformations.

\section{B. Magnetic field perpendicular to one of the spins}

We proceeded in the same way for $\vec{h}=h_{x} \hat{x}$, ranging from zero to $\left\langle M_{x}\right\rangle$ saturation. The main difference with respect to the previous case is that the spin at each site 3 is perpendicular to the magnetic field and is not affected by the Zeeman coupling. The resulting magnetic phase diagram is shown in Fig. 6. For $\delta=0$, as in the case above, there is a plateau at $\left\langle M_{x}\right\rangle=$ $1 / 2$ extending up to $\delta \lesssim 0.038$, where the effective model is dominated by $\left|J_{2}^{\text {eff }}\right| \simeq \widetilde{J}_{3 d}^{\text {eff }}$ with $J_{1}^{\text {eff }} \simeq J_{3}^{\text {eff }} \simeq J_{3 d}^{\text {eff }} / 2$. At this plateau we find two possible six-site magnetic unit cells, both with no net magnetization in the $\hat{y}$ direction. The magnetic pattern is built by translation along $\{2 \vec{a}, \vec{b}\}$ or $\{2 \vec{a}, \vec{a}+\vec{b}\}$, as shown in the second line in Fig. 7.

For $0.02 \lesssim \delta$ a plateau at $1 / 3$ appears, which widens with $\delta$. At this plateau we observe two different magnetic orders, depending of the sign of $J_{1}^{\text {eff }}$. For $0.02 \lesssim \delta \lesssim 0.06$, the $J_{2}^{\text {eff }}$ coupling dominates and $J_{1}^{\text {eff }}$ is positive. We find a nine-site star-shaped unit cell containing one of the patterns shown in the third line in Fig. 7: there are clear spin orientations for sites 1 and 2, but not for site 3, the orientations of which are completely or partially random (random orientation is represented by a purple square). For $0.06 \lesssim \delta, J_{2}^{\text {eff }}$ and $J_{3}^{\text {eff }}$ dominate and $J_{1}^{\text {eff }}$ turns negative. The transition is signaled by a clear widening of the plateau in Fig. 6 . The consequence is that the magnetic configurations no longer satisfy the kagome ice rules. At this regime a 18-site magnetic unit cell is uniquely determined, as shown in the first line in Fig. 8.

For the $\left\langle M_{x}\right\rangle=0$ plateau we have a similar situation depending on the value of $\delta$. the transition around $\delta \simeq 0.06$, where $J_{1}^{\text {eff }}=0$, is noticed as a narrowing of the plateau. Again, for $\delta \lesssim 0.06$ there are two possible nine-site star-shaped magnetic unit cells, sketched in the bottom line in Fig. 7. For $0.06 \lesssim \delta$ (negative $J_{1}^{\text {eff }}$ ) the magnetic unit cell consists of 12 


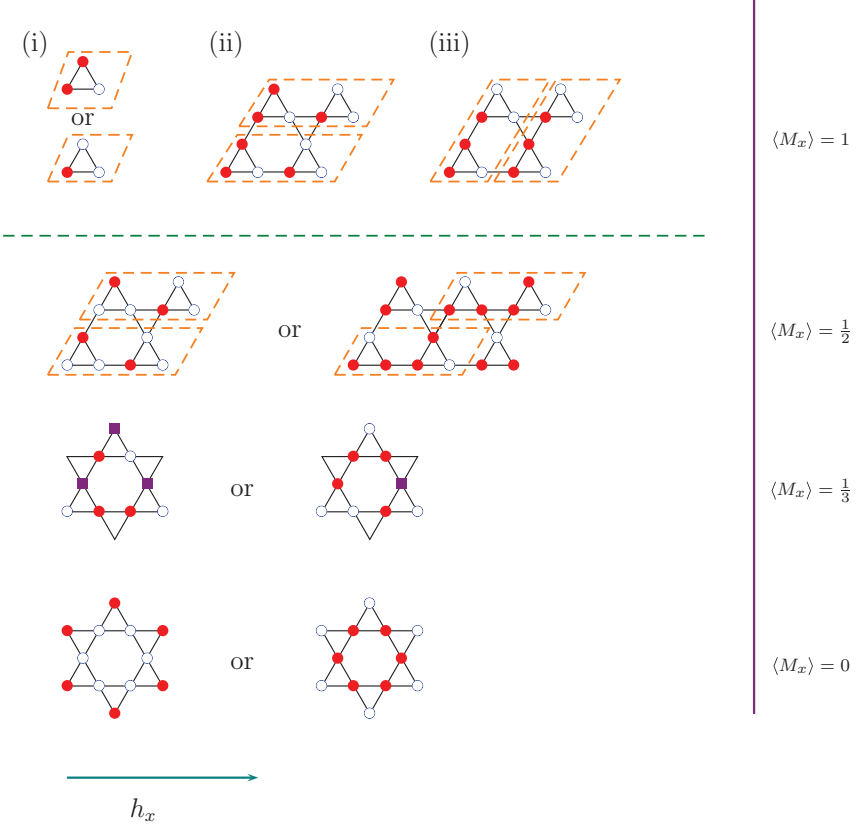

FIG. 7. (Color online) Magnetic unit cells in different magnetization plateaus for $h_{y}=0$ in the small- $\delta$ regime. Going from bottom to top, we show magnetic orders at the various plateaus present in the magnetic phase diagram shown in Fig. 6. Purple squares indicate random spin orientation.

sites with two possible arrangements, as shown in Fig. 8. From these cells, the magnetic tiling is obtained by translation along $\{2 \vec{a}, 2 \vec{b}\}$.

Finally, in the saturation configuration (Fig. 7), spins at sites 1 and 2 are aligned with the magnetic field (i.e., $\sigma_{1}=+1$ and $\sigma_{2}=-1$ ). The remaining site 3 forms a triangular lattice with anisotropic couplings, $J_{3 d}^{\text {eff }}$ along horizontal bonds and $J_{3}^{\text {eff }}$ along the others. The observed magnetic orderings in this sublattice, shown in Fig. 7 (i, ii, iii), depend on $\delta$. For $0 \leqslant \delta<0.005, J_{3}^{\text {eff }}$ is negative and dominates $\left(\left|J_{3}^{\text {eff }}\right|>J_{3 d}^{\text {eff }}\right)$, giving rise to a ferromagnetic ordering; the magnetic unit cell is indicated by (i). This phase spontaneously breaks $\mathbb{Z}_{2}$ symmetry through a net magnetization in the $\hat{y}$ direction
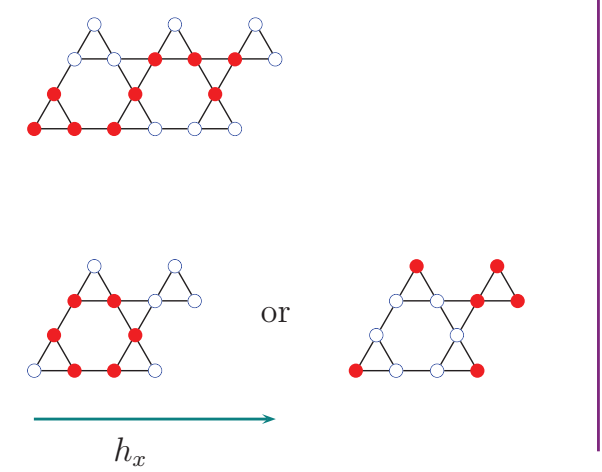

$\left\langle M_{x}\right\rangle=\frac{1}{3}$

FIG. 8. (Color online) Magnetic unit cells in the magnetization plateaus for $h_{y}=0$ in the large- $\delta$ regime. (with a temperature-driven phase transition in the Ising universality class). Then, for $0.005 \leqslant \delta<0.045, J_{3 d}^{\text {eff }}>0$ dominates $\left(\left|J_{3}^{\text {eff }}\right|<J_{3 d}^{\text {eff }}\right)$, producing antiferromagnetic order along horizontal bonds; the corresponding ordering is indicated by (ii). Finally, for $\delta \geqslant 0.045, J_{3}^{\text {eff }}$ is positive and dominates $\left(0<J_{3 d}^{\text {eff }}<J_{3}^{\text {eff }}\right)$, producing antiferromagnetic order along nonhorizontal bonds; the magnetic pattern is marked by (iii).

\section{CONCLUSIONS}

We have studied the effects of lattice deformations on the planar kagome ice with nearest-neighbor exchange and long-range dipolar interactions. We have integrated out the phonon degrees of freedom and we have kept the induced effective couplings up to third neighbors. Dipolar interactions have been truncated at the same order. On the effective Ising model we have performed Monte Carlo simulations with an external magnetic field parallel to one of the spins $\left(\vec{h}=h_{y} \hat{y}\right)$ or perpendicular to it $\left(\vec{h}=h_{x} \hat{x}\right)$. We have found several plateaus in the magnetization curves, depending on the effects of the deformations $\delta$ (which reflects the strength of the spin-phonon coupling).

In the first case and for small spin-phonon coupling, plateaus at 0 and $1 / 2$ of saturation appear. As this coupling increases, the $1 / 2$ plateau splits into a $1 / 3$ and a $2 / 3$ plateau. This situation persists until the deformations change the sign of the nearest-neighbor effective interaction, which no longer induces frustration; thus the kagome ice rules are not obeyed. In this strongly coupled regime a plethora of plateaus appear. In all these plateaus different ordered structures show up which can be easily characterized by small magnetic unit cells.

In the second case, since one out of every three spins is decoupled from the magnetic field, the situation is much simpler. There are again plateaus at 0 and $1 / 2$ for small $\delta$, and the $1 / 2$ plateau turns into a $1 / 3$ plateau as the spin-phonon coupling is increased.

In connection to the experiments that motivated the present work, ${ }^{15}$ one should notice that the regime of interest is that of small kagome plane-field components, $h_{x}$ and/or $h_{y}$. We find that in this regime, both phase diagrams show the same transitions from $M=0$ to $M=1 / 2$ for a small deformation effect $\delta$ and from $M=0$ to $M=1 / 3$ for larger $\delta$. The relevance of these results to the experiments remains to be analyzed, in particular, by considering a more realistic model including out-of-plane components of Ising spins. This will be discussed elsewhere.

\section{ACKNOWLEDGMENTS}

The authors especially thank S. Grigera and R. Borzi for communicating and discussing their results. This work was partially supported by CONICET (PIP 1691) and ANPCyT (PICT 1426).

\section{APPENDIX}

We provide here some technical details on the construction of the effective Ising Hamiltonian in Eq. (12). We start with the spin Hamiltonian in Eq. (1), including first-neighbors 
exchange $J_{0}$ and dipolar interactions characterized by firstneighbors strength $D$. We then include lattice degrees of freedom $\vec{u}_{i}$ in Eq. (2), with elastic energy

$$
\sum_{i} \frac{K}{2}\left(\vec{u}_{i}\right)^{2}
$$

The interaction of lattice degrees of freedom with spins is included by a first-order expansion of (a) an assumed isotropic dependence of the exchange coupling on the distance between lattice sites and (b) the $1 / r^{3}$ dependence in the dipolar terms.

The expansion of $J\left(r_{i j}\right)$ in Eq. (4) provides spin-phonon interaction terms

$$
-\alpha J_{0} \sum_{\langle i j\rangle^{(1)}} \hat{r}_{i j} \cdot\left(\vec{u}_{j}-\vec{u}_{i}\right) \vec{S}_{i} \cdot \vec{S}_{j}
$$

while the variation of $1 / r^{3}$ in the dipolar interactions provides

$$
\begin{aligned}
& D a^{3} \sum_{i \neq j} \frac{1}{\left(r_{i j}^{0}\right)^{4}}\left[-3 \vec{u}_{i} \cdot \hat{r}_{i j}\left(\vec{S}_{i} \cdot \vec{S}_{j}\right)\right. \\
& \quad+15 \vec{u}_{i} \cdot \hat{r}_{i j}\left(\vec{S}_{i} \cdot \hat{r}_{i j}\right)\left(\vec{S}_{j} \cdot \hat{r}_{i j}\right) \\
& \left.\quad-3\left(\vec{u}_{i} \cdot \vec{S}_{i}\right)\left(\vec{S}_{j} \cdot \hat{r}_{i j}\right)-3\left(\vec{u}_{i} \cdot \vec{S}_{j}\right)\left(\vec{S}_{i} \cdot \hat{r}_{i j}\right)\right] .
\end{aligned}
$$

All of these terms, linear in $\vec{u}_{i}$, are collected in Eq. (5) as a sum over lattice sites

$$
\sum_{i} \vec{u}_{i} \cdot\left(\sum_{j \neq i} \vec{F}_{i j}\right)
$$

where for any pair of sites $j \neq i, \vec{F}_{i j}$ contains geometric information and it is quadratic in the Ising variables $\sigma_{i} \sigma_{j}$, parameterizing spins as $\vec{S}_{i}=S \sigma_{i} \hat{e}_{i}$, where $S$ is the spin magnitude and $\hat{e}_{i}$ the local reference direction. The geometry for first- and second-neighbor sites, shown in Fig. 1, leads to the explicit expression in Eqs. (7) and (8). As stated in the main text, upon integrating the variables $\vec{u}_{i}$, one arrives to the effective Hamiltonian in Eq. (10). Finally, retaining terms with $\vec{F}_{i j}$ relating only nearest-neighbor sites produces the effective Hamiltonian in Eq. (12).

The values of the various parameters in the simulations of our simplified model have been chosen in accordance with data taken from $\mathrm{Dy}_{2} \mathrm{Ti}_{2} \mathrm{O}_{7}$ pyrochlore. ${ }^{1}$ In particular, $D \approx 1.41 \mathrm{~K}$ and $J_{0} \approx 3.72 K$, implying $d=\frac{D}{J} \approx 0.38$, which is close to the value $d \approx \frac{1}{3}$ used throughout Sec. III.

To estimate the spin-phonon coupling $\lambda$ we use data from Ref. 28, where $\mathrm{Dy}_{2} \mathrm{Ge}_{2} \mathrm{O}_{7}$ pyrochlore under high pressure is reported; the change in Dy ion distance and the change of the exchange coupling with respect to $\mathrm{Dy}_{2} \mathrm{Ti}_{2} \mathrm{O}_{7}$ allows us to fit $\lambda \approx$ $-\left(\Delta J_{0} / J_{0}\right) /(\Delta a / a) \approx 20$. This value fulfills the condition in Eq. (11), in support of using a truncated effective Hamiltonian.
*Corresponding author: albarrac@ fisica.unlp.edu.ar

${ }^{1}$ S. T. Bramwell and M. J. P. Gingras, Science 294, 1495 (2001).

${ }^{2}$ M. J. Harris, S. T. Bramwell, D. F. McMorrow, T. Zeiske, and K. W. Godfrey, Phys. Rev. Lett. 79, 2554 (1997).

${ }^{3}$ S. T. Bramwell, M. J. Harris, B. C. den Hertog, M. J. P. Gingras, J. S. Gardner, D. F. McMorrow, A. R. Wildes, A. L. Cornelius, J. D. M. Champion, R. G. Melko, and T. Fennell, Phys. Rev. Lett. 87, 047205 (2001).

${ }^{4}$ A. P. Ramirez, A. Hayashi, R. J. Cava, R. Siddharthan, and B. S. Shastry, Nature (London) 399, 333 (1999).

${ }^{5}$ T. Fennell, O. A. Petrenko, G. Balakrishnan, S. T. Bramwell, J. D. M. Champion, B. Fåk, M. J. Harris, and D. M. Paul, Appl. Phys. A 74, 889 (2002).

${ }^{6}$ A. A. Zvyagin, Low Temperature Physics/Fizika Nizkikh Temperatur 39, 1159 (2013).

${ }^{7}$ M. J. P. Gingras, Introduction to Frustrated Magnetism, Springer Series in Solid-State Sciences (Springer, Heidelberg, 2011), Chap. 12.

${ }^{8}$ J. D. Bernal and R. H. Fowler, J. Chem. Phys. 1, 515 (1933).

${ }^{9}$ L. Pauling, J. Am. Chem. Soc. 57, 2680 (1935).

${ }^{10}$ A. L. Cornelius and J. S. Gardner, Phys. Rev. B 64, 060406 (2001).

${ }^{11}$ H. Fukazawa, R. G. Melko, R. Higashinaka, Y. Maeno, and M. J. P. Gingras, Phys. Rev. B 65, 054410 (2002).

${ }^{12}$ R. Moessner and S. L. Sondhi, Phys. Rev. B 68, 064411 (2003).

${ }^{13}$ S. V. Isakov, K. S. Raman, R. Moessner, and S. L. Sondhi, Phys. Rev. B 70, 104418 (2004).

${ }^{14}$ H. Sato, K. Matsuhira, T. Sakakibara, T. Tayama, Z. Hiroi, and S. Takagi, J. Phys.: Condens. Matter 19, 145272 (2007).
${ }^{15}$ S. A. Grigera, D. Slovinsky, R. D. Borzi, A. S. Gibbs, R. Higashinaka, Y. Maeno, and T. S. Grigera (unpublished).

${ }^{16}$ T. Yavors'Kii, T. Fennell, M. J. P. Gingras, and S. T. Bramwell, Phys. Rev. Lett. 101, 037204 (2008).

${ }^{17}$ R. D. Borzi (unpublished).

${ }^{18}$ A. Keren and J. S. Gardner, Phys. Rev. Lett. 87, 177201 (2001).

${ }^{19}$ R. Moessner and S. L. Sondhi, Phys. Rev. B 68, 064411 (2003).

${ }^{20}$ T. Vekua, D. C. Cabra, A. Dobry, C. Gazza, and D. Poilblanc, Phys. Rev. Lett. 96, 117205 (2006).

${ }^{21}$ A. S. Wills, R. Ballou, and C. Lacroix, Phys. Rev. B 66, 144407 (2002).

${ }^{22}$ G. Möller and R. Moessner, Phys. Rev. B 80, 140409 (2009).

${ }^{23}$ C. Nisoli, R. Moessner, and P. Schiffer, Rev. Mod. Phys. 85, 1473 (2013).

${ }^{24}$ D. L. Bergman, R. Shindou, G. A. Fiete, and L. Balents, Phys. Rev. B 74, 134409 (2006).

${ }^{25}$ K. Penc, N. Shannon, and H. Shiba, Phys. Rev. Lett. 93, 197203 (2004).

${ }^{26}$ F. Wang and A. Vishwanath, Phys. Rev. Lett. 100, 077201 (2008).

${ }^{27}$ Notice that if the bond phonon model is used for elastic degrees of freedom, at this stage one observes that the product $\vec{F}_{i j} \cdot \vec{F}_{i j}$ leads to a trivial effective Hamiltonian where all Ising variables are squared.

${ }^{28}$ H. D. Zhou, S. T. Bramwell, J. G. Cheng, C. R. Wiebe, G. Li, L. Balicas, J. A. Bloxsom, H. J. Silverstein, J. S. Zhou, J. B. Goodenough, and J. S. Gardner, Nat. Commun. 2, 478 (2011).

${ }^{29}$ D. Landau and K. Binder, A Guide to Monte Carlo Simulations in Statistical Physics (Cambridge University Press, New York, 2000).

${ }^{30}$ S. Kirkpatrick, C. D. Gelatt, and M. P. Vecchi, Science 220, 671 (1983). 